\title{
Chapter
}

\section{Theories of nanoparticle and nanostructure formation in liquid phase}

\author{
Ali Karatutlu ${ }^{* * * *}$, Ahmed Barhoum ${ }^{\ddagger, \S}$ and Andrei Sapelkin ${ }^{\dagger}$ \\ *UNAM-National Nanotechnology Research Center, Bilkent University, Ankara, Turkey; **The Institute of Materials \\ Science and Nanotechnology, Bilkent University, Ankara, Turkey; ${ }^{\dagger}$ Centre for Condensed Matter and Materials Physics, \\ Queen Mary University of London, London, United Kingdom; ${ }^{*}$ Vrije Universiteit Brussel (VUB), Brussels, Belgium; \\ ${ }^{\S}$ Helwan University, Helwan, Cairo, Egypt
}

\section{INTRODUCTION}

Nanoparticles (NPs) and nanostructured materials exhibit shape- and sizedependent properties that are desired for a wide variety of applications, such as catalysis, sensing, drug delivery, energy production, and storage $[1,2]$. In view of this, it is essential to produce well-defined NPs and nanostructures with desired characteristics, to understand their formation and growth mechanisms, and to define the critical size below which they act differently from bulk materials in order to develop synthetic strategies [3]. For example, quantum dots (below $20 \mathrm{~nm}$ ) are mainly single nanocrystals characterized by a single-domain crystalline lattice without grain boundaries $[4,5]$. These tiny individual crystals differ drastically from bulk polycrystalline materials [6]. They are characterized by a very small size and a very high surface-to-volume ratio, and therefore have unique optoelectronic properties [7]. In fact, existing investigations indicated that ordered polycrystalline particles are preferably formed at high supersaturations, where rapid nucleation generates many NPs, which subsequently tend to aggregate randomly at high NP concentrations. Single crystals, such as quantum dots, form at low supersaturations. The reduction of the supersaturation to a level at which primary NPs are still formed in solution yields mesocrystals [8].

The liquid-phase synthesis of NPs and nanostructures can be classified into (1) top-down approaches, where bulk materials are etched in an aqueous solution for producing NPs or nanostructures, and (2) bottom-up approaches, where atoms/molecules are held together via nucleation, assembling, growth, 
and crystallization. In general, tiny NP "clusters" are gathered together to form NPs, and then the NPs are assembled to form nanostructures. In bottom-up approaches, cluster formation and nucleation are the first steps in NP formation, and they are the most crucial steps in controlling the crystal structure, size, shape, and surface characteristics of NPs [9]. Nucleation is the process whereby nuclei (seeds) act as templates for crystal growth. Nucleation may happen in the solution homogeneously or heterogeneously. Homogeneous nucleation occurs when nuclei form uniformly within the parent phase, whereas heterogeneous nucleation forms on a solid support (nucleating surface), such as container surfaces, impurities, grain boundaries, or dislocations [10]. In general, heterogeneous nucleation occurs much more easily than homogenous nucleation, since a stable nucleating surface is already present.

The fundamental phenomena involved in crystallization from solutions via nucleation and growth is still a hot topic [11]. Recently, significant efforts have been directed to understanding the mechanism of NP formation in solutions [12-14]. In particular, attention has been directed to study the wide varieties of additives and their influences on prenucleation clusters, nucleation, crystallization, nanocrystal assembly, and thus morphologies.

The importance of NPs and nanostructures in many fields of science and technology has spurred a high level of research activity and led to the formulation of well-established theories for nucleation and crystal growth. This chapter highlights the different theories, such as classical nucleation theory (CNT), La Mer's nucleation and growth mechanisms, the two-step nucleation and growth mechanism, and the prenucleation cluster (PNC) mechanism. Subsequently in situ experimental characterization for NP formation, including nucleation and growth processes, will be highlighted, and what occurs within the different processes outlined.

\section{CLASSICAL NUCLEATION THEORY}

As the precipitation process includes the nucleation and growth stages, it would have been more convenient to start first with the classical theory of nucleation. In order to begin the nucleation process, there should be a seed substance, called the nuclei, obtained from the consumption of monomers. Then, the crystallization process occurs between the nuclei and the solution. Thus, nuclei are considered to be the building blocks of crystals. According to CNT, nuclei is created as a new phase within the bulk liquid upon the aggregation of clusters. The nucleation of a new phase is largely determined by the nucleation work. The aggregation of the cluster may result in the spontaneous formation of the critical nucleation of the new phase. For this reason, the 
nucleation work is the energy barrier (critical energy of cluster formation) of the nucleation. The critical nucleus "new phase" is regarded as a liquid drop with a sharp interface (a dividing surface) that separates the new and parent bulk phases.

The thermodynamic part of CNT was developed by J.W. Gibbs. In 1878, Gibbs published his monumental work, On the Equilibrium of Heterogeneous Substances. Later, Gibbs extended his work and introduced a method to derive an exact formula for $\Delta G$ in the nucleation of a new phase in the bulk parent phase. Based on his assumptions, the free energy $\Delta G(r)$ of a spherical particle with a radius $r$ is the summation of the free energy due to the new surface creation and the free energy due to new volume creation. In other words, the free energy of this critical nuclei relative to the liquid phase is the sum of two terms: a positive contribution from the surface free energy $\Delta G_{S}$ and a negative contribution from the bulk free energy $\Delta G_{V}$ difference between the supersaturated vapor and the liquid. The surface free energy results from the reversible work used in forming the interface between the new phase and the liquid phase. Naturally, the size of the critical nucleation, the critical energy, the phase transition probability, and the nucleation rate can be obtained by the determined nucleation work. Thus, the overall free energy, $\Delta G(r)$ is expressed [7,15] as shown in Eq. (20.1):

$$
\Delta G(r)=\Delta G_{S}+\Delta G_{V}=4 \pi r^{2} \sigma-\frac{4}{3 V} \pi r^{3} k_{B} T \ln (S)
$$

where $\sigma$ is the surface free energy per unit area, $V$ is the molecular volume of the nucle $i$ in the crystal, $k_{B}$ is the Boltzmann constant, $T$ is the temperature in Kelvin, and $S$ is the saturation ratio. The interfacial free energy is also known as the surface free energy, which is used for the free energy difference per molecule between the bulk and the surface contributions. The wet state synthesis of NPs is held inside a solution; thus, it is correct to use the interfacial free energy instead. The surface free energy can be used interchangeably when the formation of NPs is performed inside the vacuum environment.

In order to initiate the nucleation process, the solution becomes supersaturated either at high temperature/pressure or by chemical reactants. This means the saturation ratio will be larger than 1 and $\Delta G(r)$ becomes positive when the particle radius reaches the critical size $r_{c r i t}$, where the maximum free energy is obtained (Fig. 20.1). This maximum free energy known as the activation energy or the thermodynamic energy barrier, $G^{*}$ is required for the nucleation. The critical size can also be considered to be a metastable state $\left(\frac{\partial \Delta G^{*}}{\partial r}=0\right.$ and $\left.\frac{\partial^{2} \Delta G^{*}}{\partial r^{2}}<0\right)$. Thus, any infinitesimal negative change in the particle radius will lead to the nucleus dissolution, as any infinitesimal 


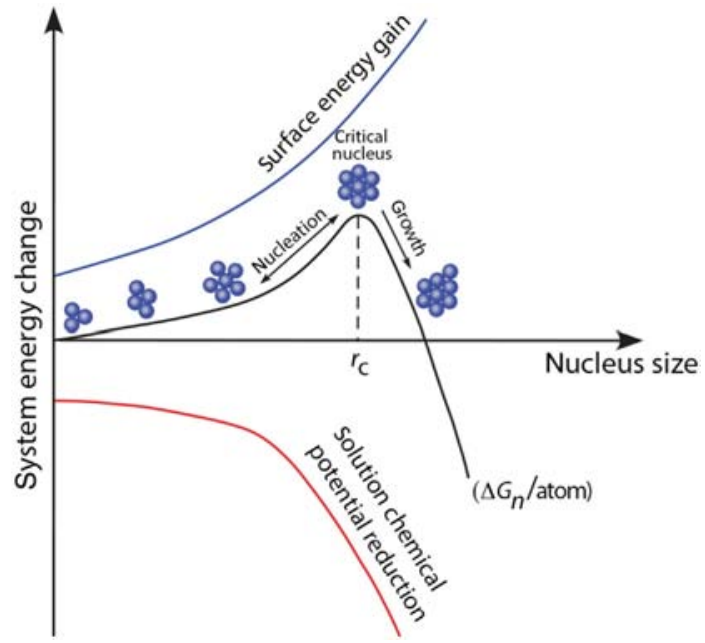

FIGURE 20.1 Schematic illustration of the formation of nanoparticles, represented using classical nucleation theory as a function of the nanoparticle (NP) radius $r$. A

metastable state is reached when the radius of the NPs reaches the critical radius value $r$ at which NPs are formed. Any infinitesimal reduction in size will lead to the dissolution of NPs back into the solution, as any infinitesimal rise in size over $r$, will lead to the continuous growth of NPs. (Source: Reproduced with permission from Ref. [16]. Copyright 2013, Mineralogical Society of America.)

positive size variation will cause a continuous growth. The critical size is expressed [7] as shown in Eq. (20.2):

$$
r_{c}=\frac{2 V \sigma}{3 k_{B} T \ln (S)}
$$

The nucleation process is divided into three different categories: homogeneous nucleation, heterogeneous nucleation, and secondary nucleation. In homogeneous nucleation, NPs are formed upon reaching the top of the energy barrier; here, for the sake of simplicity, homogeneous nucleation is defined as the nucleation of the NPs with a spherical shape grown inside the solution. In heterogeneous nucleation, there are two different elements (molecular species) needed, and one is grown on top of another. NPs are formed where the foreign (impure) atoms, such as those at the surface of the reaction vessel, exist (see Refs. $[15,17]$ to read further about heterogeneous and secondary nucleation).

The rate of homogeneous nucleation $J$ in a supersaturated solution is usually defined as the number of nuclei (aggregation events) nucleated per cubic centimeter per second. The rate of nucleation in homogeneous nucleation, $J$ is expressed [14] as shown in Eq. (20.3):

$$
J=A \cdot e^{\frac{-E_{A}}{k_{B} T}} \cdot e^{\frac{\Delta G^{*}}{k_{B} T}}
$$


Here, the first exponential term is related to the kinetic barriers, including overall activation energy $E_{\mathrm{A}}$, and the second exponential term corresponds to the thermodynamic barrier.

\section{LA MER'S NUCLEATION AND GROWTH MECHANISMS}

This section will deal briefly with some of useful concepts, including burst nucleation, diffusion-limited growth, and Ostwald (slow) ripening, which is used to control the size distribution of NPs. La Mer's diagram (Fig. 20.2) is generally used for this purpose [18,19], in order to explain schematically that the nucleation and the growth processes are separated from each other over time. In other words, the prenucleation process produces the burst of nuclei required for monodispersity that occurs in a very short time, followed by the relatively slow growth of NPs with respect to the nucleation process.

The nucleation process is complete after the critical supersaturation limit is reached. At this point the growth process is initiated. In the course of the growth of colloidal NPs, monomers are consumed and their concentrations are depleted over time. There are two main growth mechanisms, including diffusion-limited growth (also called Ostwald ripening) and reaction-controlled growth. To understand the wet-state synthesis of NPs, understanding the growth process is important. In small NPs, the surface

(A)

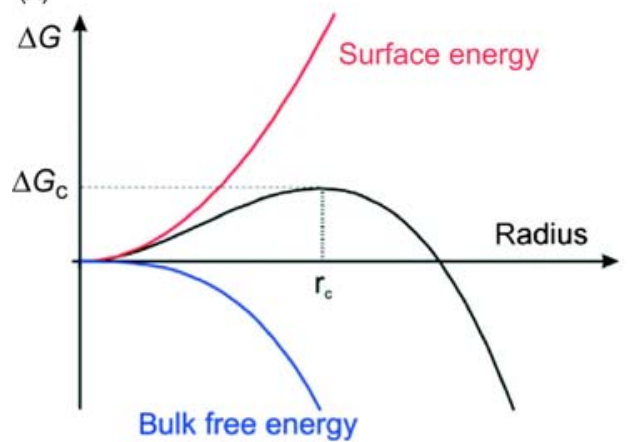

(B)

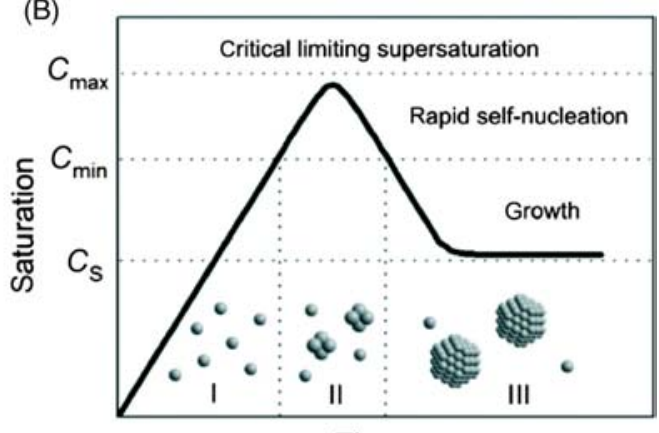

Time

FIGURE 20.2 (A) The dependence of the cluster free energy $\Delta G$ on the cluster radius $r$, according to classical nucleation theory. The curve has a maximum free energy $\Delta G$ at a critical cluster size $r_{c^{\prime}}$ which defines the first stable particles — the nuclei. (B) La Mer's diagram schematically represents that the nucleation and growth processes are separated temporally. The peak shows the critical supersaturation level. The (theoretical) qualitative curve describes the monomer concentration as a function of time. (Source: Ref. [20]. Published by the Royal Society of (hemistry.) 
free energy part is the dominating portion in the formation of total free energy. This means that when relatively larger NPs are formed, the surface energy contribution is reduced. The monomers react at the surface of NPs for the growth of the colloidal NPs, when monomers are diffused from the solution to the surface of the NPs. This diffusion process is known as Ostwald ripening process.

Colloidal NPs suspended in liquids move randomly due to Brownian motion. In such cases, liquids surrounding the NP surface can be assumed stationary with a fluid thickness $\delta$ (Fig. 20.3). Here, the monomers bulk concentration $C_{B}$, the monomers concentration at the interface $C_{i}$, and the solubility of the NP as a function of its radius $r$ are also represented. When the NP radius $r$ with a density $d$ increases, the ratio of $r / \delta$ decreases [21].

Following the studies $[21,22]$, the total flux of monomers traversing a spherical volume with a radius $x$ inside the diffusion layer is defined by Fick's first law, as shown in Eq. (20.4):

$$
J=4 \pi x^{2} D \frac{d c}{d x}
$$

where $D$ and $C$ are the diffusion coefficient and the monomer concentration at distance $x$, respectively. Here, $J$ does not change over the distance $x$, as the diffusion layer is assumed to be stationary. When $C$ is integrated with respect to $x$, then the flux is obtained as shown in Eq. (20.5):

$$
J=\frac{4 \pi D r(r+\delta)}{\delta}\left(C_{B}-C_{i}\right)
$$

(A)

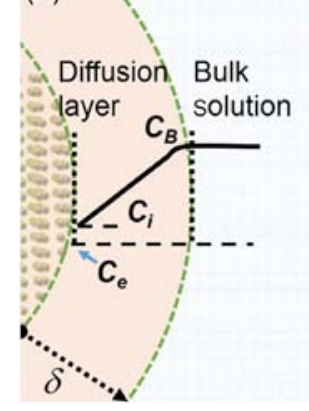

(B)

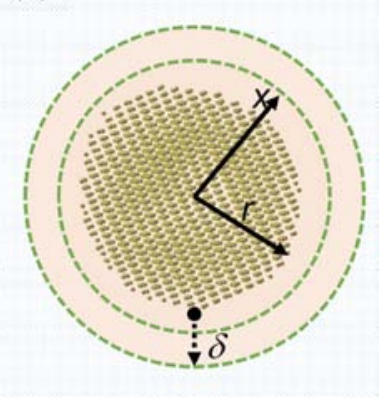

FIGURE 20.3 (A) The solute concentration profile is given in a diffusion layer. (B) A diffusion layer is schematically represented when the diffusion layer surrounds a spherical nanoparticle. (Source: Modified with permission from Ref. [22]. Copyright 1987, Elsevier.) 
After the reaction is initiated at the NP surface via consuming monomers, the diffusion rate is considered to be a first-order reaction, and thus is written proportionally to a rate constant $k$ as shown in Eq. (20.6):

$$
J=4 \pi r^{2} k\left(C_{i}-C_{e}\right)
$$

When Eqs. (20.5) and (20.6) are written together, it is expressed as shown in Eq. (20.7):

$$
\frac{\left(C_{i}-C_{e}\right)}{\left(C_{B}-C_{i}\right)}=\frac{D}{k r}\left(1+\frac{r}{\delta}\right)
$$

In diffusion-limited growth, $D \ll k r$ and the condition of $C_{i}=C_{e}$ is obtained. Therefore, changing $C_{i}$ in Eq. (20.5) to $C_{e}$ results in the following, shown by Eq. (20.8):

$$
J=\frac{4 \pi D r(r+\delta)}{\delta}\left(C_{B}-C_{e}\right)
$$

The variable $J$ can also be expressed in terms of the molar volume of the solid $V_{m}$ and the gradient of $r$ with respect to $t$, as shown by Eq. (20.9):

$$
J=\frac{4 \pi r^{2}}{V_{m}} \frac{d r}{d t}
$$

Using Eqs. (20.8) and (20.9), $\frac{d r}{d t}$ can be expressed as shown by Eq. (20.10):

$$
\frac{d r}{d t}=D V_{m}\left(\frac{r+\delta}{r \delta}\right)\left(C_{B}-C_{e}\right)=D V_{m}\left(\frac{1}{r}+\frac{1}{\delta}\right)\left(C_{B}-C_{e}\right)
$$

Eq. (20.10) states that when the size of the NPs increases, $d r / d t$ decreases. That means the size distribution would be narrower for relatively larger NPs if $\left(C_{B}-C_{e}\right)$ is kept constant. From Eq. (20.10), the relation between the standard deviation of the size distribution $\Delta r$ and the mean radius of NPs $\tilde{r}$ can be obtained, as shown by Eq. (20.11):

$$
\Delta r=\frac{\tilde{r}+\delta}{\tilde{r}}=1+\frac{\delta}{\tilde{r}}
$$

After this point, reaction-limited growth is considered. Reaction-limited growth yields, $D \gg k r$ in Eq. (20.7), and consequently $C_{B} \cong C_{i}$. Eq. (20.12) follows:

$$
\frac{d r}{d t}=k V_{m}\left(C_{B}-C_{e}\right)
$$


Eq. (20.12) means that the growth rate is controlled by the surface reaction of monomers. This also means that $d r / d t$ does not depend on the size of the NPs and that the standard deviation of the size distribution $\Delta r$ is constant. This causes a reduction in the relative standard deviation $\frac{\tilde{r}}{r}$ when NP growth occurs.

Fig. 20.4A shows the solute concentration profile for the general-growth mode, the diffusion-limited growth mode, and the reaction-controlled growth mode. The reaction-controlled growth mode is divided into two parts: polynuclear layer growth (PLG) and mononuclear layer growth (MLG) [23] (Fig. 20.4B). During PLG, the fast nucleation on the surface can limit the two-dimensional growth range of each nuclear step on a nanocrystal. If the growth on the NP surface is enormously fast relative to the nucleation rate, a single nucleus will initiate the surface being covered by a new layer. This is called MLG.

For a colloidal NP, the ratio between $r$ and $\delta$ is considered to be around 0.25; thus the diffusion-limited growth rate given in Eq. (20.10) becomes Eq. (20.13):

$$
\frac{d r}{d t} \cong 1.25 D V_{m} \frac{\left(C_{B}-C_{e}\right)}{r}
$$

Eq. (20.13) tells that in diffusion-limited growth, the growth rate is inversely proportional to the NP radius. However, in Eq. (20.12) it seems that the growth rate does not depend directly on the NP radius. Therefore, this can be used as a criterion to distinguish between growth modes. This criterion
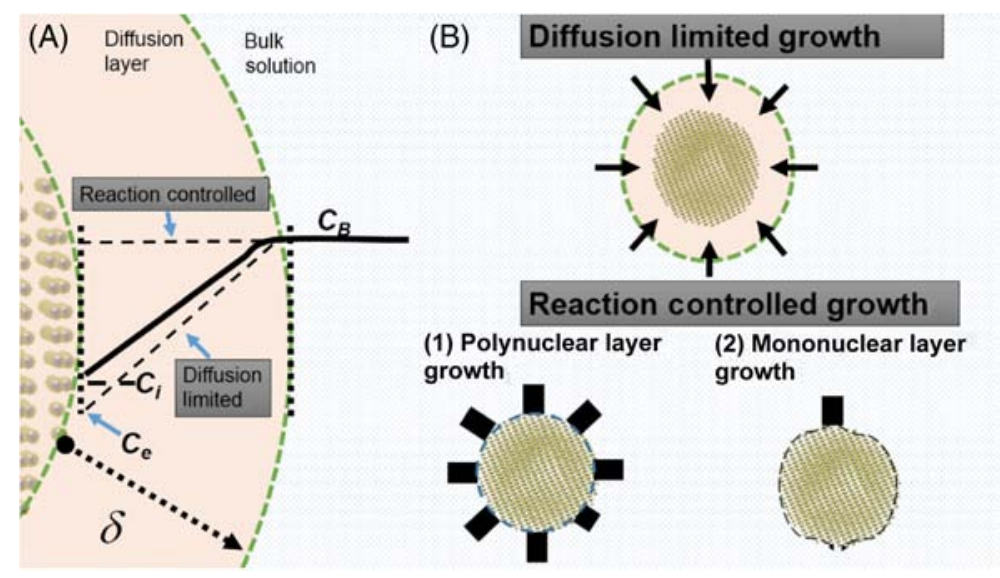

GIGURE 20.4 (A) The solute concentration profile for diffusion-limited growth and reactioncontrolled growth. (B) Schematics of three different growth models are demonstrated. (Source: Modified with permission from Ref. [21]. Copyright 2001, Elsevier.) 
is known as the size dependence of the growth rate. Likewise, various other criteria have been outlined [21] to discriminate between diffusion-limited growth and reaction-controlled growth. These are (1) magnitude of the growth rate, (2) activation energy of the growth rate, (3) evolution of the size-distribution width, (4) effect of the particle number of concentration, (5) solubility dependence of the growth rate, and (6) chronomal analysis. Any of these criteria can be used in experimental investigations to understand the characteristics of NP formation.

Before discussing this, in addition to the classical theoretical studies mentioned up to now, we will include some of the mathematical models developed particularly for the liquid-phase synthesized NPs to describe their nucleation and growth processes $[24,25]$. In these models, the NP radius is accepted to be much smaller than the diffusion layer thickness, which leads to a rearrangement of the Gibbs-Thomson equation for smaller particle sizes. This is achieved by considering diffusion-limited growth with an infinite diffusion layer $(\delta=\infty)$, as shown by Eq. (20.14):

$$
C_{e}=C_{\infty} e \frac{2 \sigma V_{m}}{r R T}
$$

where $C_{\infty}, \sigma, R$, and $T$ are the solubility of the bulk solid, the specific surface energy, the gas constant, and the absolute temperature, respectively.

For NPs, $\frac{2 \sigma V_{m}}{r R T} \ll 1$ is approximated and Eq. (20.14) becomes Eq. (20.15):

$$
C_{e} \cong C_{\infty}\left(1+\frac{2 \sigma V_{m}}{r R T}\right)
$$

$C_{B}$ can similarly be rearranged and written as Eq. (20.16):

$$
C_{e} \cong C_{\infty}\left(1+\frac{2 \sigma V_{m}}{r R T}\right)
$$

Therefore, based on the abovementioned assumptions, the growth rate of NPs is expressed as shown in Eq. (20.17):

$$
\frac{d r}{d t} \cong \frac{K}{r}\left(\frac{1}{r^{*}}-\frac{1}{r}\right)
$$

where $K=\frac{2 \sigma D V_{m}^{2} C_{\infty}}{R T}$.

One of the first models of liquid-phase synthesized NPs was proposed by Mantzaris [24]. The validity of this model was verified in extreme cases, where the Damköhler (Da) number was accepted to be 0 for reaction-controlled growth, and for diffusion-limited growth, $\infty$ obeyed the Lifshitz-Slyozov-Wagner theory in terms of the asymptotic behavior of the system. In other words, the 
Da number yields the relative magnitude of surface reaction with respect to diffusion. Higher-order terms in the Taylor series expansion of the exponential term in the Gibbs-Thompson equation were included to be able to take into account of very small particle sizes. This is led to a mathematical model producing a size distribution of NPs and the effect of the continuous injection of the monomers into the reaction chamber based on their size, which ignores the nucleation part but justifies NP growth and Ostwald ripening.

In the second model, Robb and Privman developed a first-time model to quantify the burst nucleation together with the diffusion-limited growth [25]. Here, below the critical radius, a thermalization effect was introduced for burst nucleation. The model can be applied to any spherical NP system in a supersaturated solution. Due to colloidal nature of NPs, aggregation effect was found to be trivial in this model. Their model shows that NP size increases proportionally with time $t$ and that relative width shrinks proportionally with $t^{-1 / 2}$.

These models assume that the NPs are spherical NPs, but that for experimentation purposes they could still be utilized to gain some insights. Thus, the models must be reevaluated for liquid-phase synthesized NPs with various shapes (e.g., see Ref. [26] for the anisotropic growth of NPs).

The following sections will provide an overview of additional nucleation and growth mechanisms, including (1) the two-step nucleation and growth mechanism, and (2) the PNC mechanism.

\section{THE TWO-STEP NUCLEATION AND GROWTH MECHANISM}

The two-step mechanism starts in the presence of disordered liquid or amorphous metastable clusters in the homogeneous solutions existing prior to nucleation. Metastable clusters have been observed for several protein systems and for $\mathrm{CaCO}_{3}$ solutions. However, it is likely that not all solutions would support the formation of clusters with properties allowing the nucleation of crystals in them. The two-step nucleation mechanism is used to explain crystal nucleation systems in solutions, including (1) nucleation rates that are orders of magnitude lower than theoretical predictions, (2) nucleation kinetic dependencies at increasing supersaturation levels, and (3) the role of heterogeneous substrates in the nucleation process and polymorph selection [27]. The two-step nucleation mechanism also provides powerful tools for controlling the nucleation process by varying the solution thermodynamic parameters [27]. Fig. 20.5 shows a schematic illustration of the two-step mechanism for the nucleation of crystals. The sequential pathway (Fig. 20.5B) corresponds to the formation of a droplet of dense liquid followed by the formation of a crystalline nucleus inside this droplet. If the dense liquid is stable with respect to the dilute solution, the nucleation of 
crystals occurs inside macroscopic droplets in this phase. A crystal nucleus may form inside the cluster (Fig. 20.5B). A far more common case is when the dense liquid is not stable and has a higher free energy than the dilute solution. In these cases, the dense liquid is contained in metastable clusters (intriguing objects in their own right) and crystal nucleation occurs within the clusters.

In the two-step nucleation and growth mechanism [28], nucleation and growth processes are considered to explain quantitatively the time dependence of the formation of NPs. The nucleation process is a slow and

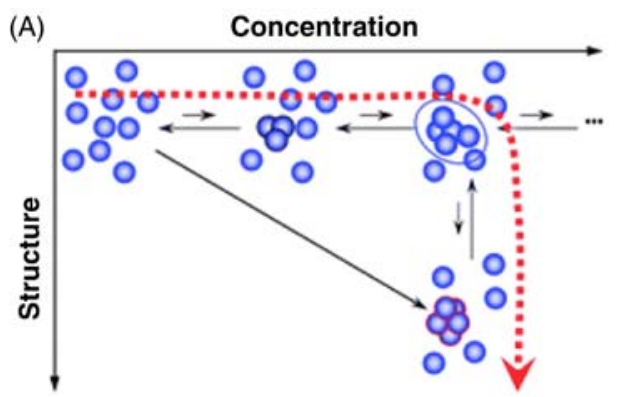

(B)

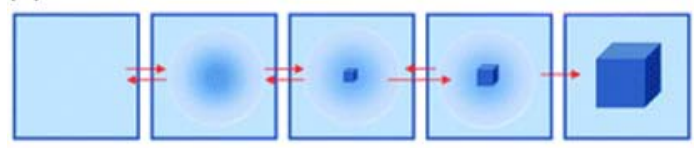

(C)

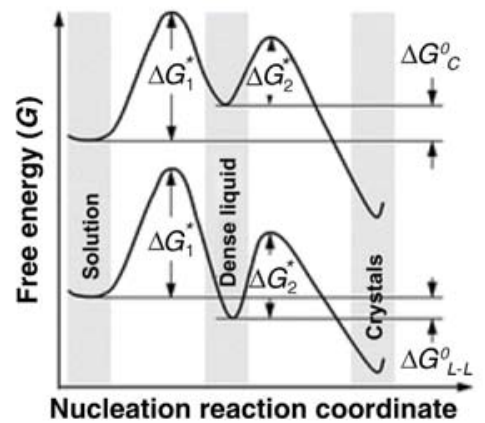

FIGURE 20.5 The two-step mechanism of the nucleation of crystals. (A) Microscopic viewpoint of the (concentration vs. structure) plane. (B) Macroscopic viewpoint of events along the dotted line in (A) is illustrated. (C) The free-energy $\Delta G$ along two possible pathways is given for nucleation of crystals from the solution. The standard free energy of formation of the dense liquid phase is denoted by $\Delta G_{L-L}^{0}<0$. If the dense liquid is unstable and $\Delta G_{L-L}^{0}>0$, the dense liquid is present as mesoscopic clusters, $\Delta G_{L-L}^{0}<0$ is changed with $\Delta G_{c}^{0}$, and the upper curve applies; however, if the dense liquid is stable and $\Delta G_{L-L}^{0}<0$, the lower curve applies. The barrier for formation of a cluster of dense liquid is denoted by $\Delta G_{1}^{*}$ whereas the barrier for a structure fluctuation leading to an ordered cluster is denoted by $\Delta G_{2}^{*}$. (Source: Reproduced from Ref. [27] with permission from the Royal Society of Chemistry.) 
continuous process, where the pseudoelementary step A $\rightarrow$ B is achieved at rate constant $k_{1}$. This process is followed by a growth process (called autocatalytic surface growth) at rate constant $k_{2}$. In this growth process, the pseudoelementary step $\mathrm{A}+\mathrm{B} \rightarrow \mathrm{AB}$ occurs. This method, including the experiments carried out for the formation of $\operatorname{Ir}(0)$ NPs, makes four predictions about the design of the formation processes of NPs, particularly for transition metals. These predictions are as follows: (1) adding more metal precursor, and (2) checking the ratio of $k_{2} / k_{1}$ can increase the size of NPs but also can lead to little to no new nucleation; at the same time (3) closed shell, magic-number nanoclusters might be preferred due to their intrinsically greater thermodynamic stability, which would provide in return (4) the possibility of adjusting the size distributions of the nanoclusters close to the magic numbers (e.g., $\mathrm{M}_{13}, \mathrm{M}_{55}, \mathrm{M}_{147}, \mathrm{M}_{309}, \mathrm{M}_{561}, \mathrm{M}_{923}$, etc.). This method was also thought to fit well for other transition metals, including Pt, Ru, and $\mathrm{Rh}$ [29]. Despite the fact that the nucleation mechanism in this method is different than that described in the classical nucleation mechanism section, the nucleation step here also takes into account critical size.

\section{THE PRENUCLEATION CLUSTER MECHANISM}

The five most important features of the PNC mechanism [14,30,31] include the following: (1) PNCs are formed from constituent atoms, molecules, or ions and can also contain other chemical species. (2) PNCs are small solutes in their lowest energy state (i.e., chemically stable with dissolved ions distributed in them), and are therefore considered to have no phase boundary between the clusters and the surrounding solution. (3) PNCs are initiated during the process of phase separation, when they act as molecular precursors for the phase to be nucleated from the solution. (4) PNCs show very dynamic characteristics and can change their configuration and rearrange themselves in the order of picoseconds. (5) PNCs bear structural motifs (or intermediate amorphous phases) that substitute for or resemble base-forming crystals.

This method was initially studied using a titration-based experimental set up for $\mathrm{CaCO}_{3}$ crystallization $[30,31]$ and further tested using nanoclusters in silica-rich solutions of $\mathrm{CaCO}_{3}$ [32]. The same approach has recently been tested for barium sulphate $\left(\mathrm{BaSO}_{4}\right)$ mineral precipitation [33], which was studied together with $\mathrm{CaCO}_{3}$ precipitation. Barhoum et al. combined several strategies using the so-called seed-mediated hot-injection route to control the growth of $\mathrm{Ag}$ NPs on $\mathrm{CaCO}_{3}$ PNCs [10]. A hypothetical mechanism was discussed, based on the principles of the classical and nonclassical theories of nucleation (Fig. 20.6).

The concept of PNCs cannot fully explain the nature of $\mathrm{Au}_{m} \mathrm{~S}_{n}^{-} \cdot p \mathrm{H}_{2} \mathrm{O}$ species [34]. According to theory, PNCs are stable solutes with a "molecular" character 


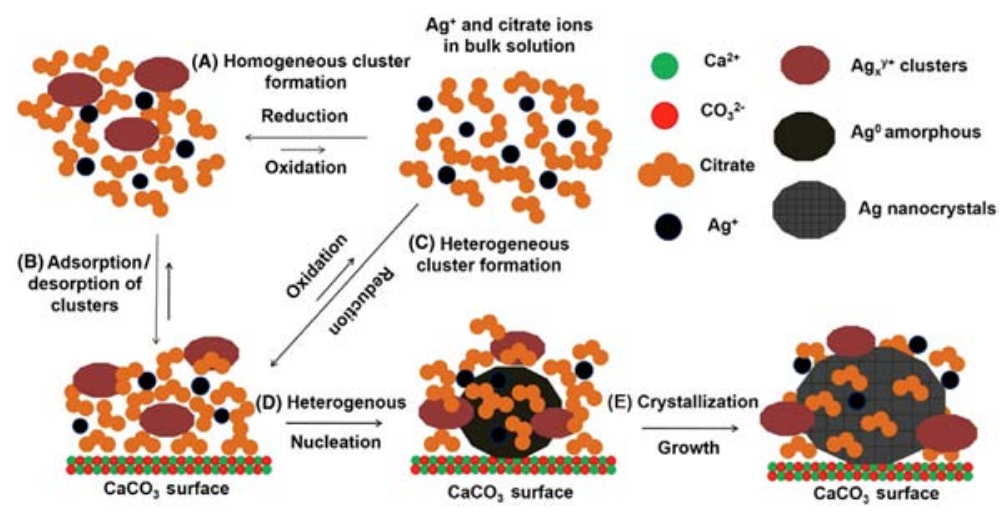

FIGURE 20.6 The different steps in the seed-mediated formation of tiny Ag nanoclusters on the $\mathrm{CaCO}_{3} \mathrm{NC}$ surface, which are covered by a monolayer of citrate ions (not to scale). (A) Homogenous cluster formation: the complexation and redox reaction of $\mathrm{Ag}^{+}$and citrate ions in the solution induces the homogeneous formation of tiny clusters $\left(\mathrm{Ag}_{x}^{y+}\right)$ stabilized by citrate ions. (B) Adsorption/desorption of $\left(\mathrm{Ag}_{x}^{y+}\right)$ clusters on the $\mathrm{CaCO}_{3} \mathrm{NC}$ surface. (C) Heterogeneous formation of $\left(\mathrm{Ag}_{x}^{y+}\right)$ clusters on the $\mathrm{CaCO}_{3} \mathrm{NC}$ surface, possibly facilitated by the citrate monolayer adsorbed on the $\mathrm{CaCO}_{3} \mathrm{NC}$ surface; further citrate electron transfer reduction of $\mathrm{Ag}^{+}$to metallic silver $\mathrm{Ag}^{0}$ on the surface. (D) Heterogeneous nucleation, densification, and formation of metastable amorphous $\mathrm{Ag}^{0}$ nuclei on the $\mathrm{CaCO}_{3}$ surface. (E) Growth and crystallization of amorphous $\mathrm{Ag}^{0}$ nuclei, forming spherical Ag NCs. The further growth of the Ag NCs is controlled by the adsorption of citrate ions. (Source: Reproduced with permission from Ref. [10]. Copyright American Chemical Society.)

that emerge in aqueous solutions prior to nucleation, which differs from ion pairs and liquid and amorphous clusters. Their formation is believed to occur via a negligible free energy barrier, and only the final transformation of PNCs into a crystalline nucleus requires some activation energy (Fig. 20.7C). Current research shows that stable solutes, liquid clusters, and droplets can coexist in the same reaction medium. The $\mathrm{Au}_{m} \mathrm{~S}_{n}^{-} \cdot p \mathrm{H}_{2} \mathrm{O}$ solutes arise rapidly at room temperature, so the activation energy is low (Fig. 20.7D). Then, the fluid intermediates (referred to as "liquid clusters" after Refs. [27,35-37] accumulating the NCs via density fluctuations emerge and grow to $\sim 12 \mathrm{~nm}$ within about $30 \mathrm{~min}$, remaining in the solution for many hours [34].

In the following section, some of the in situ experimental approaches evaluating the mechanisms of NPs formation will be mentioned.

\section{IN SITU CHARACTERIZATION TECHNIQUES}

Advanced in situ and ex situ characterization techniques have allowed the elucidation of nucleation and growth mechanisms of NPs and nanostructures: UV-Vis spectroscopy (such as quantum, and small-angle X-ray scattering, or SAXS) is used to determine the particle size of tiny NPs, and a liquid cell in the transmission electron microscopy (TEM) technique is used to further 


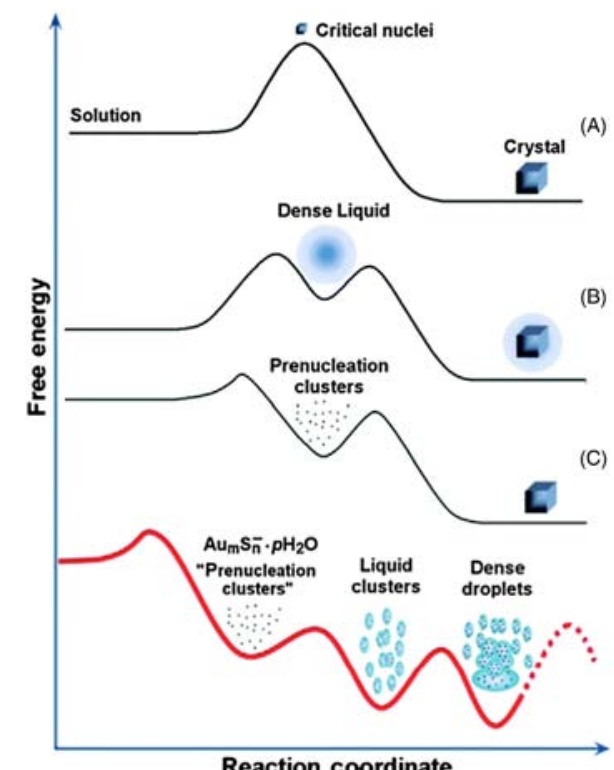

FIGURE 20.7 Illustration of the different stages and relevant free energy profiles for four mechanisms. (A) Classical mechanism, (B) two-step nucleation, (C) stable PNC formation, and (D) the mechanism proposed here for the formation of gold and gold sulfide. (Source: Reproduced from Ref. [34] with permission from the PCCP Owner Societies.)

probe NPs in situ and study how NPs grow in solution. For example, Brazeau and Jones have studied nucleation and the growth of these quantum dots by UV-Vis is spectroscopy [38]. Zheng et al. have directly observed the growth of individual Pt nanocrystals in solution by using a liquid cell that operates inside a special TEM [12]. Teng has investigated the growth of pure calcite crystals by atomic force microscopy [39]. Pontoni et al. carried out SAXS experiments and discovered that the most likely growth mechanism of silica NPs is coalescence, which is diffusion controlled after the initial nucleation phase [40]. Other experimental studies investigating the mechanisms of NPs formation have been highlighted as well [7,12,13,19]. Experimental approaches are important to determine whether there is a single burst of nucleation followed by a pure growth phase. They could also demonstrate the size-dependent growth rate theory, as well as the effect of the capping agent on the nucleation and growth processes. It is noteworthy to mention that investigations into the nucleation and the growth kinetics of $\mathrm{CdX}(\mathrm{X}=\mathrm{S}, \mathrm{Se}$, Te) NPs seem to be popular in the field. There are also a few studies, mostly using the classical theories mentioned above, accounting for the nucleation 
and growth of colloidal NPs in solutions, such as the nucleation of Ag NPs [41] and the growth of Au NPs and ZnO NPs [19]. Some of these experiments are discussed in chronological order next.

In one of the earlier studies, Peng et al. published a paper titled "Kinetics of IIVI and III-V Colloidal Semiconductor Nanocrystal Growth: 'Focusing' of Size Distributions" [42]. Here, the term focusing refers to the observation that the initial nucleation period was much less than the time required for the growth process in which the size distributions are decreased further. They showed that when the NP diameter was increased from 2.1 to $3.3 \mathrm{~nm}$, the corresponding size distribution was reduced from $20 \%$ to $7.7 \%$. The size evolution data were extracted from their photoluminescence spectroscopy studies of the aliquots of the samples CdSe and InP nanocrystals when the reaction was sustained. This study shows that the burst of nucleation and size focusing were held with a high growth rate in the early stage of the growth period. The authors followed a size-dependent growth rate (estimated from Eq. (20.17)) using the GibbsThompson equation [22] (Eq. (20.14)) was used in the case of NPs.

Similarly to the above-mentioned method, Bullen and Mulvaney showed an example of estimating the nucleation kinetics of CdSe nanocrystals in octadecene using UV-Vis absorption spectroscopy studies to determine the size of CdSe nanocrystals [43]. In the study done by Yu and his team, the particle concentration within the each aliquot was obtained from the extinction coefficient, which was previously derived from the NP size, estimated using the corresponding exciton energy of each sample [44]. They found that the size of CdSe nanocrystals quickly increased over a reaction time of $300 \mathrm{~s}$, but that CdSe nucle $i$ were lowered when the capping agent (oleic acid) concentration was increased (Fig. 20.8). On the other hand, the size distribution after 5 min increased slowly, as expected from Ostwald ripening.

A different but also important study [45] also demonstrated the nucleation and the growth mechanism of the CdSe NPs using in situ fluorescence detected extended X-ray absorption spectroscopy (EXAFS) [46] and ex situ UV-Vis absorption techniques. The data collected via these two techniques were taken under the same conditions. In situ EXAFS measurements were taken at the Se $\mathrm{K}$-edge to investigate the change in the local structure during the reaction solution flowing with constant speed at $7.6 \mathrm{~mm} / \mathrm{s}$ inside a heated Kapton tube for a limited time $(<8.1 \mathrm{~s})$. The EXAFS study demonstrated that the nucleation process yielding transformation from the Se-P phase (from TOP-Se solution) to the Se-Cd phase arising from CdSe nuclei in the course of growing CdSe nanocrystals was completed within few seconds. After the EXAFS data were recorded, the aliquots from the solutions prepared under the same conditions were prepared for UV-Vis absorption spectroscopy measurements in order to 
612 CHAPTER 20 Theories of nanoparticle and nanostructure formation in liquid phase
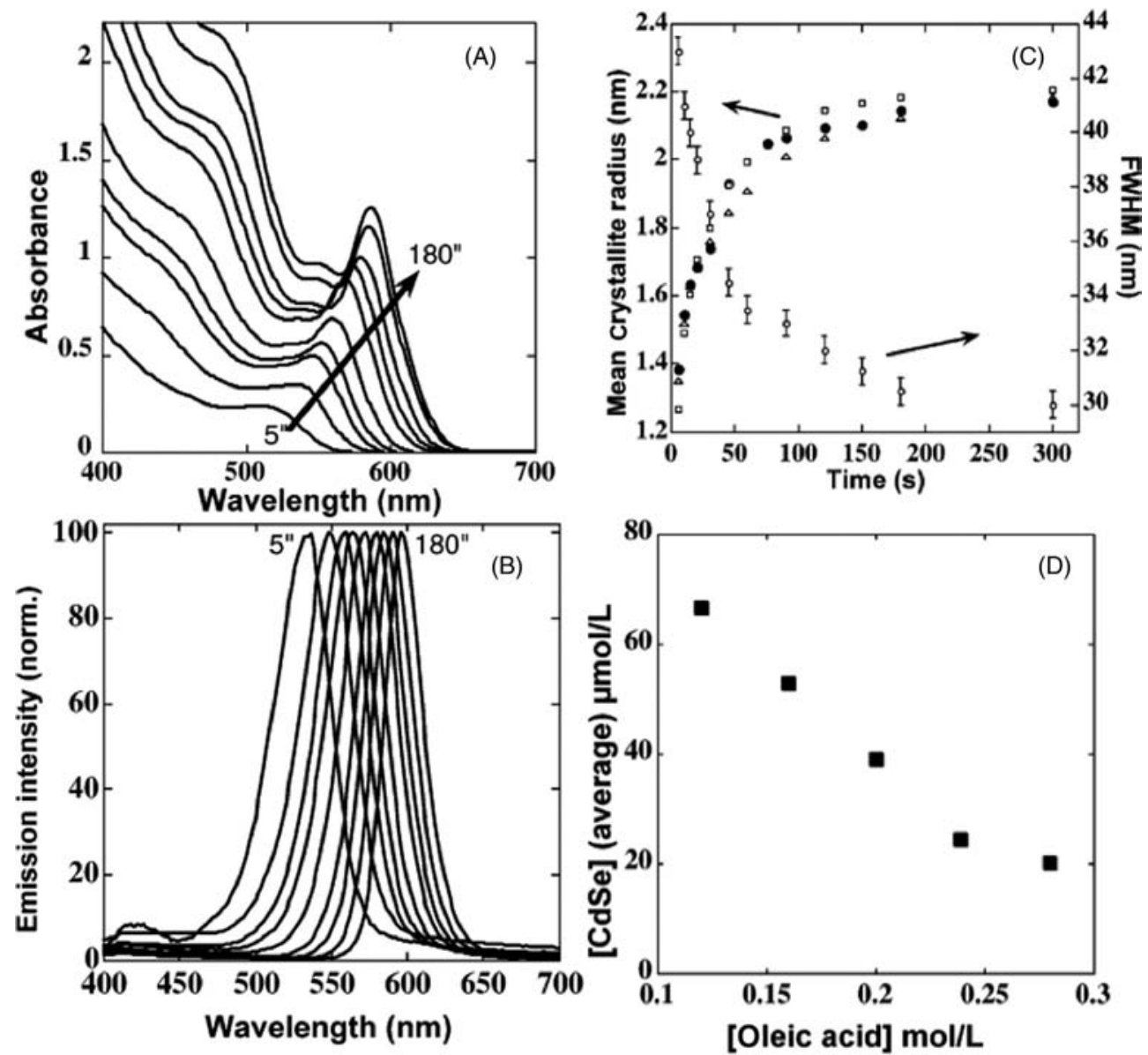

FIGURE 20.8 (A) The UV-Vis absorption spectra, and (B) the photoluminescence (PL) spectra during the reaction over $180 \mathrm{~s}$ are demonstrated. (C) The size and the full width at half maximum (FWHM) (open circles) estimated from (A) are shown versus the reaction time for the CdSe nanocrystals. (D) The CdSe nuclei concentration is lowered when increasing the oleic acid concentration. (Source: Reproduced with permission from Ref. [43]. Copyright 2004, American Chemical Society.)

confirm the nucleation and the growth of CdSe nanocrystals [43]. Therefore, particularly in situ, the EXAFS technique is valuable to probe quick processes, such as nucleation, inside a solution and accessible at various synchrotron sources with a high signal-to-noise ratio.

Another interesting study [12] on the growth mechanism of colloidal platinum NPs was done using in situ TEM with subnanometer resolution. This study demonstrated that the growth of the NPs takes place due to monomer consumption on the surface or by multiple particle coalescence events. A broad 
size width was initially obtained, which led NPs to grow with monodisperse size distribution. In the above-mentioned theoretical studies, the growth process of NPs is considered to be a continuous process. Yet the growth mechanism of the platinum NPs was unexpectedly found to include a punctuated growth after a coalescence event and carry on the diffusion-controlled Ostwald ripening process $\left(d^{3} \sim t\right)$. This means that there is a relaxation time after each coalescence event. When the relaxation time passes, the growth continues. The effect of surfactant was also found to play an important role in the growth process. When the surfactant concentrations were reduced, different platinum morphologies, such as nanocrystal foils and dendrites, were noticed.

A similar approach using in situ TEM was applied for the nucleation and growth kinetics of Au on Pt icosahedral NPs [47]. The data obtained from this in situ TEM study for the growth kinetics of the Au/Pt NPs were studied based on the LSW theory. Eq. (20.18), which appears to be an improved form of Eq. (20.17), was utilized with a consideration of growth and diffusion simultaneously after the study [19]:

$$
\frac{d r}{d t}=\frac{k}{r}\left(\frac{1}{D}-\frac{1}{k_{D} r}\right)\left(\frac{1}{r^{*}}-\frac{1}{r}\right)
$$

where $k=\frac{2 \sigma D V_{m}^{2} C_{\infty}}{R T} . D$ is the diffusion coefficient and $k_{D}$ is the rate constant for deposition.

A quantitative analysis of nucleation, diffusion, and conformal coating of $\mathrm{Au}$ on a Pt particle is shown in Fig. 20.9. For this analysis, the six distances were categorized into two groups: three distances between the two opposite corners $\left(D_{\mathrm{c}}\right)$ and three distances between the two parallel opposite sides $\left(D_{\mathrm{s}}\right)$. The distance and time (Fig. 20.9A) was measured for the growth of Au on Pt NPs. The TEM micrographs were recorded at a rate of $0.1-0.5$ frame/s for $D_{\mathrm{c}}$ and $D_{\mathrm{s}}$. In this sequence, the recording process was initiated in $5 \mathrm{~min}$, just after the stock solution was hosted into the liquid cell. Three distinctive regions were obtained for the entire sequence. $D_{\mathrm{c}}$ and $D_{\mathrm{s}}$ were increased at a rate of $0.013 \mathrm{~nm} / \mathrm{s}$ and $0.010 \mathrm{~nm} / \mathrm{s}$, respectively, after the observation started in Region I. However, the growth was approximately $200 \mathrm{~s}$ behind the rate obtained in $D_{c}$. In Region II, $D_{\mathrm{c}}$ was reduced to a rate of $\sim 0.017 \mathrm{~nm} / \mathrm{s}$, whereas $D_{\mathrm{s}}$ sustained its increase at the similar rate of $\sim 0.012 \mathrm{~nm} / \mathrm{s}$. The growth rates of both $D_{\mathrm{c}}$ and $D_{\mathrm{s}}$ were observed to increase to $\sim 0.07 \mathrm{~nm} / \mathrm{s}$ after the flow initiated at $1500 \mathrm{~s}$. Therefore, the growth rate in both $D_{\mathrm{c}}$ and $D_{\mathrm{s}}$ was observed to be similar when the Au-coated icosahedron was formed, without a difference between the facets.

This study demonstrates the growth kinetics involving the processes (1) deposit of Au occurs on corner sites of Pt icosahedral NPs, (2) Au atoms are 


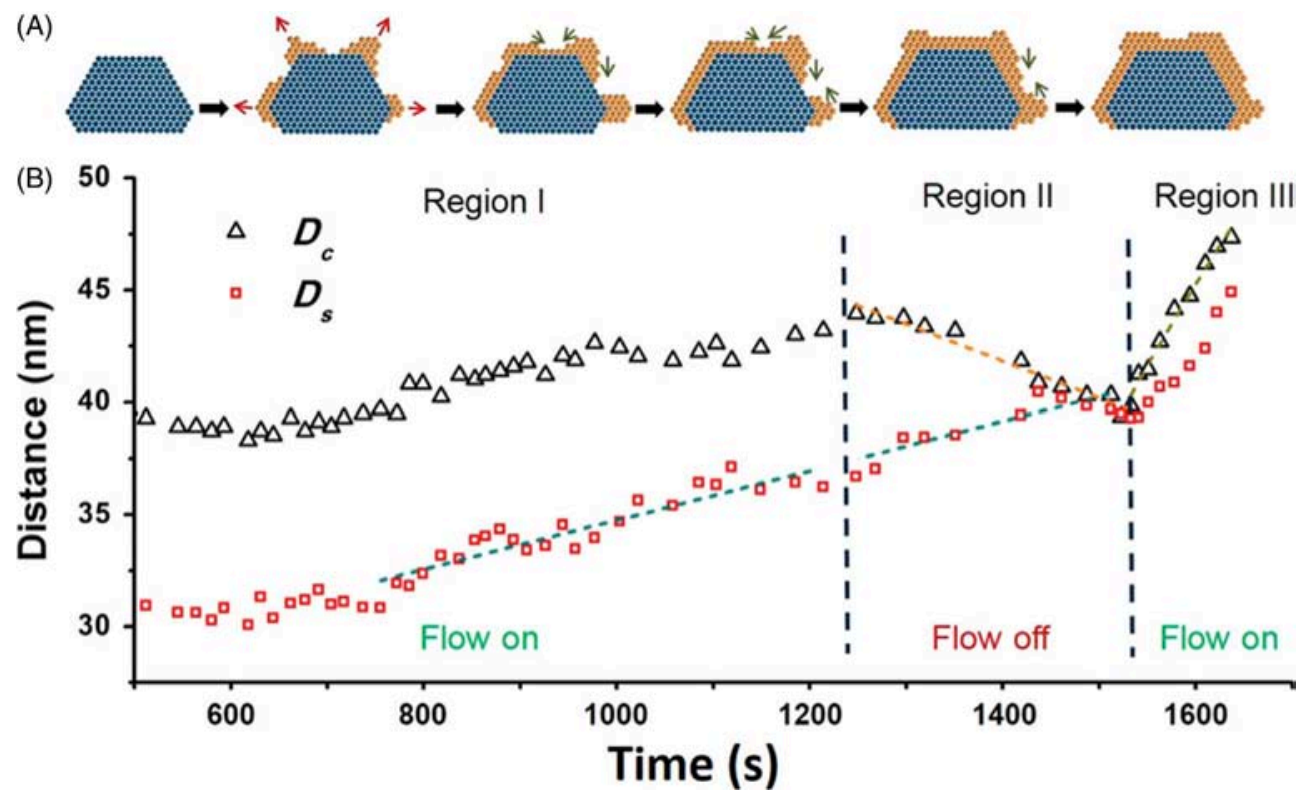

FIGURE 20.9 (A) The processes of Au depositing on a Pt icosahedral nanocrystal is schematically demonstrated. (B) The nucleation and growth of Au on Pt nanocrystals are quantitatively analyzed. (Source: Reproduced with permission from Ref. [47]. Copyright 2015, American Chemical Society.)

diffused from the corners to terraces and edges, and (3) Au/Pt (core/shell) NPs are formed by the subsequent layer-by-layer growth.

More importantly, using different ligands in the preparation of NPs can lead to drastically different behaviors. These two different circumstances were shown for the nucleation and growth of Au NPs with alkanoic acid and alkylamine using in situ SAXS/wide-angle X-ray scattering and UV-Vis spectroscopy [48]. Here, the data were obtained with the benefit of the synchrotron radiation for a time resolution in the order of ms. As mentioned earlier, the time resolution is particularly important because Au NPs are formed within a few seconds. Prior to this study, it was also helpful to know the absorption bands of metal complexes, including $\mathrm{Au}(\mathrm{III}), \mathrm{Au}(\mathrm{I})$, and $\mathrm{Au}(0)$. Thus, in situ UVVis absorption spectroscopy was temporally used to monitor qualitatively the different phases in the increase of absorption bands from the Au(III)-based ions $(\lambda \approx 400 \mathrm{~nm}$ ) [49] into the surface plasmon absorption band of Au NPs $\left(\lambda_{p}=544 \mathrm{~nm}\right)$. The reduction of the Au(III) salt was found to be much faster and continuous in the case of the amine, whereas the increase in the case of the acid was shown to include a break in the increase of plasmon intensity.

The results estimated for the two different ligands from the fit of the SAXS data using the Levenberg-Marquardt algorithm are shown in Fig. 20.10 
(A)
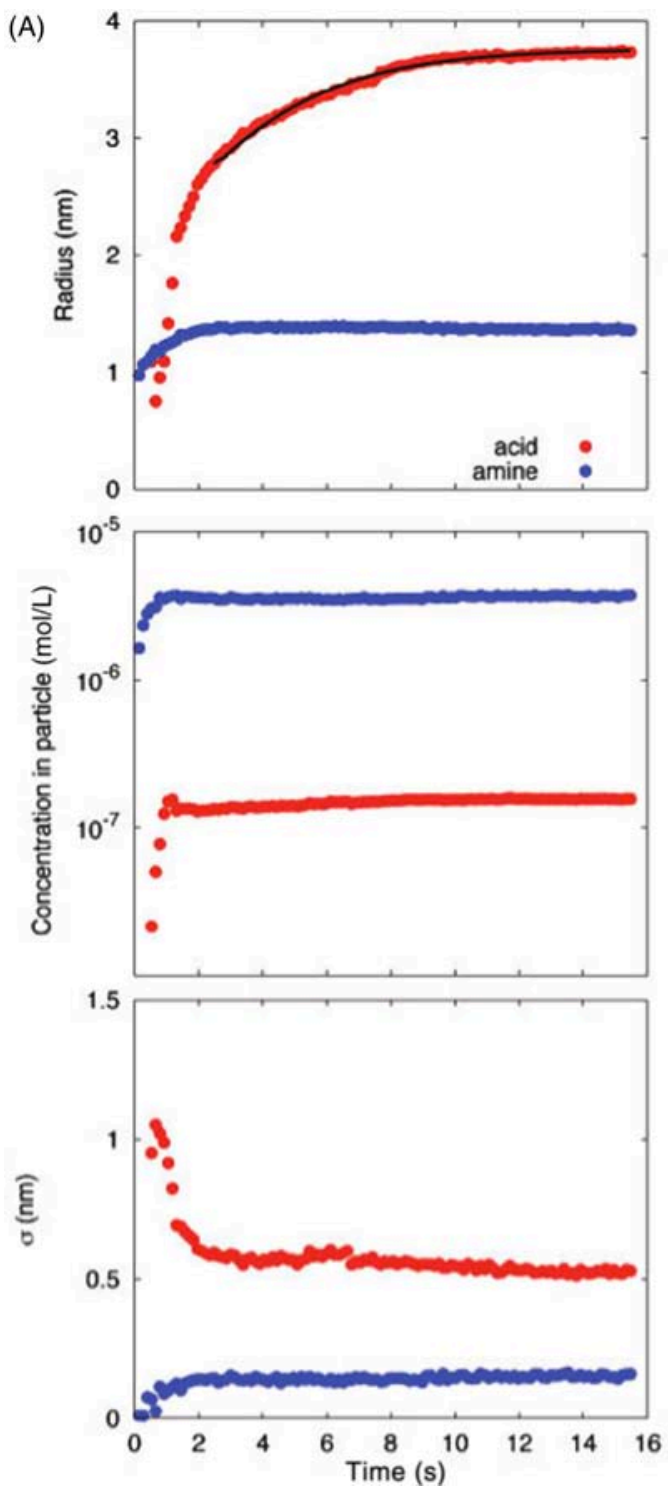

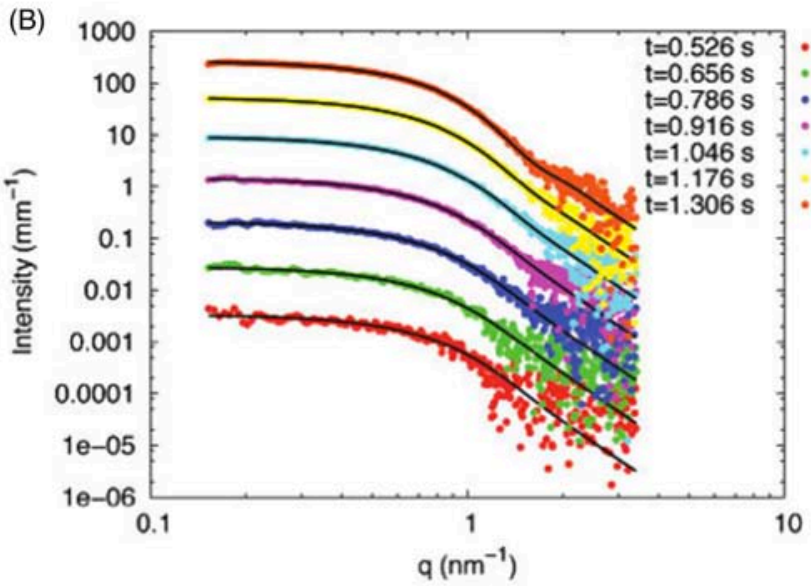

(C)

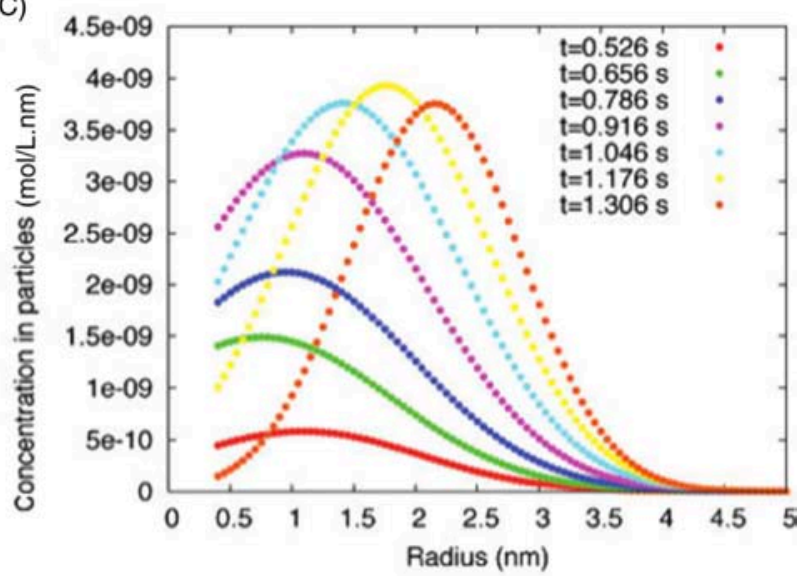

FIGURE 20.10 Radius, concentration, and size distribution from the SAXS fitting results. (A) The results of the fit of the SAXS patterns are presented as a function of time. For the two different ligands, the concentration of particles $\left(n / N_{A}\right)$, the center of the Gaussian distribution, and the $\sigma$ parameter are designated. (B) SAXS patterns for the first instants of the reaction and the corresponding fits for an acid ligand are shown. (C) Corresponding size distribution is given for the SAXS patterns presented in (B). (Source: Reproduced with permission from Ref. [48]. Copyright 2007, American (hemical Society.) 
for three parameters, including radius, concentration, and size distribution. This shows that the chemical nature of the ligand determines the final radius. For example, $r=3.5 \mathrm{~nm}$ for the acid, and $r=1.4 \mathrm{~nm}$ for the amine. The nucleation rate was found to be relatively larger for the amine ligands $\left(1.48 \times 10^{18} \mathrm{~L}^{-1} \mathrm{~s}^{-1}\right)$ when compared to the acid ligands $\left(5.99 \times 10^{16} / \mathrm{L} \mathrm{s}\right)$. This result suggests that the higher the nucleation rate yields, the smaller the final particle size.

\section{CONCLUSIONS}

In this chapter we highlighted theories of nucleation and growth mechanisms of NPs and nanostructured materials in liquid phase. CNT, La Mer's nucleation and growth mechanism, two-step nucleation and growth mechanism, prenuclear cluster mechanisms, and finally, in situ experimental characterization techniques, were discussed. In comparison to two-step nucleation mechanism, CNT can overestimate several measured kinetic curves, such as nucleation rates, and does not take into account the existence of spinodal for the solution in crystal phase transition and the action of a two-step nucleation mechanism; this is due to recent advances in the nucleation and growth mechanisms of NPs and nanostructured materials. Experimental in situ characterization techniques provide an opportunity to observe the formation mechanisms of NPs directly and suggests that theoretical studies should be reconsidered for the presence of events such as an interruption in growth (the punctuated growth). Different capping agents used in the preparation of NPs are demonstrated to alter nucleation and growth kinetics; thus, changing the ligands for the formation of the very same NP system (e.g., Au NPs) should be considered, with consideration of determination of parameters such as particle size, size distribution, and growth rate.

\section{REFERENCES}

[1] K.A. Brown, S. Dayal, X. Ai, G. Rumbles, P.W. King, Controlled assembly of hydrogenase-CdTe nanocrystal hybrids for solar hydrogen production, J. Am. Chem. Soc. 132 (28) (2010) 9672-9680.

[2] C. Corot, P. Robert, J.-M. Idée, M. Port, Recent advances in iron oxide nanocrystal technology for medical imaging, Adv. Drug Deliv. Rev. 58 (14) (2006) 1471-1504.

[3] M.A. El-Sayed, Small is different: shape-, size-, and composition-dependent properties of some colloidal semiconductor nanocrystals, Acc. Chem. Res. 37 (5) (2004) 326-333.

[4] S.M. El-Sheikh, A. Barhoum, S. El-Sherbiny, F. Morsy, A.A.-H. El-Midany, H. Rahier, Preparation of superhydrophobic nanocalcite crystals using Box-Behnken design, Arab. J. Chem. (2014)doi: 10.1016/j.arabjc.2014.11.003.

[5] A. Barhoum, G. Van Assche, A.S.H. Makhlouf, H. Terryn, K. Baert, M.-P. Delplancke, H. Rahier, A green, simple chemical route for the synthesis of pure nanocalcite crystals, Cryst. Growth Des. 15 (2) (2015) 573-580. 
[6] K.T. Early, D.J. Nesbitt, Size-dependent photoionization in single CdSe/ZnS nanocrystals, Nano Lett. 13 (10) (2013) 4844-4849.

[7] C. Burda, X. Chen, R. Narayanan, M.A. El-Sayed, Chemistry and properties of nanocrystals of different shapes, Chem. Rev. 105 (4) (2005) 1025-1102.

[8] J. Fang, B. Ding, H. Gleiter, Mesocrystals: syntheses in metals and applications, Chem. Soc. Rev. 40 (11) (2011) 5347-5360.

[9] A. Barhoum, L. Van Lokeren, H. Rahier, A. Dufresne, G. Van Assche, Roles of in situ surface modification in controlling the growth and crystallization of $\mathrm{CaCO}_{3}$ nanoparticles, and their dispersion in polymeric materials, J. Mater. Sci. 50 (24) (2015) 7908-7918.

[10] A. Barhoum, M.F. Rehan, H. Rahier, M. Bechelany, G. Van Assche, Seed-mediated hot injection synthesis of tiny Ag nanocrystals on nanoscale solid supports and reaction mechanism, ACS Appl. Mater. Interfaces 8 (16) (2016) 10551-10561.

[11] F.C. Meldrum, H. Cölfen, Controlling mineral morphologies and structures in biological and synthetic systems, Chem. Rev. 108 (11) (2008) 4332-4432.

[12] H. Zheng, R.K. Smith, Y.-W. Jun, C. Kisielowski, U. Dahmen, A.P. Alivisatos, Observation of single colloidal platinum nanocrystal growth trajectories, Science 324 (5932) (2009) 1309-1312.

[13] S.G. Kwon, T. Hyeon, Formation mechanisms of uniform nanocrystals via hot-injection and heat-up methods, Small 7 (19) (2011) 2685-2702.

[14] D. Gebauer, M. Kellermeier, J.D. Gale, L. Bergström, H. Cölfen, Pre-nucleation clusters as solute precursors in crystallisation, Chem. Soc. Rev. 43 (7) (2014) 2348.

[15] J.J. DeYoreo, P.G. Vekilov, Principles of crystal nucleation and growth, Rev. Mineral Geochem. 54 (1) (2003) 57-93.

[16] H. Henry Teng, How ions and molecules organize to form crystals, Elements 9 (3) (2013) 189-194.

[17] G.D. Botsaris, Secondary nucleation-a review, in: J.W. Mullin (Ed.), Industrial Crystallization, Springer, Boston, MA, 1976, pp. 3-22.

[18] V. LaMer, R. Dinegar, Theory, production and mechanism of formation of monodispersed hydrosols, J. Am. Chem. Soc. 72 (8) (1950) 4847-4854.

[19] R. Viswanatha, D.D. Sarma, Growth of nanocrystals in solution, in: C.N.R. Rao, A. Müller, A.K. Cheetham (Eds.), Nanomaterials Chemistry: Recent Developments and New Directions, Wiley-VCH Verlag, Weinheim, Germany, 2007, pp. 139-170.

[20] J. Polte, Fundamental growth principles of colloidal metal nanoparticles - a new perspective, CrystEngComm 17 (36) (2015) 6809-6830.

[21] T. Sugimoto, General principles for the formation of monodispersed particles, Monodispersed Particles, Elsevier, New York, NY, United States, (2001) pp. 187-207.

[22] T. Sugimoto, Preparation of monodispersed colloidal particles, Adv. Colloid Interface Sci. 28 (C) (1987) 65-108.

[23] A.E. Nielsen, Kinetics of precipitation, Pergamon Press, Oxford, United Kingdom, (1964).

[24] N.V. Mantzaris, Liquid-phase synthesis of nanoparticles: particle size distribution dynamics and control, Chem. Eng. Sci. 60 (17) (2005) 4749-4770.

[25] D.T. Robb, V. Privman, Model of nanocrystal formation in solution by burst nucleation and diffusional growth, Langmuir 24 (1) (2008) 26-35.

[26] Y. Yin, A.A. Paul, Colloidal nanocrystal synthesis and the organic-inorganic interface, Nature 437 (7059) (2005) 664-670. 


\section{CHAPTER 20 Theories of nanoparticle and nanostructure formation in liquid phase}

[27] P.G. Vekilov, The two-step mechanism of nucleation of crystals in solution, Nanoscale 2 (11) (2010) 2346.

[28] M.A. Watzky, R.G. Finke, Nanocluster size-control and "magic number" investigations, experimental tests of the "living-metal polymer" concept and of mechanismbased size-control predictions leading to the syntheses of iridium $(0)$ nanoclusters centering about four sequential magic, Chem. Mater. 9 (12) (1997) 3083-3095.

[29] N.T.K. Thanh, N. Maclean, S. Mahiddine, Mechanisms of nucleation and growth of nanoparticles in solution, Chem. Rev. 114 (15) (2014) 7610-7630.

[30] D. Gebauer, A. Volkel, H. Colfen, Stable prenucleation calcium carbonate clusters, Science 322 (5909) (2008) 1819-1822.

[31] D. Gebauer, H. Cölfen, A. Verch, M. Antonietti, The multiple roles of additives in $\mathrm{CaCO}_{3}$ crystallization: a quantitative case study, Adv. Mater. 21 (4) (2009) 435-439.

[32] M. Kellermeier, D. Gebauer, E. Melero-García, M. Drechsler, Y. Talmon, L. Kienle, W. Kunz, Colloidal stabilization of calcium carbonate prenucleation clusters with silica, Adv. Funct. Mater. 22 (20) (2012) 4301-4311.

[33] C. Ruiz-Agudo, C.V. Putnis, A. Ibañez-Velasco, E. Ruiz-Agudo, A. Putnis, A potentiometric study of the performance of a commercial copolymer in the precipitation of scale forming minerals, CrystEngComm 18 (30) (2016) 5744-5753.

[34] Y. Mikhlin, A. Karacharov, M. Likhatski, T. Podlipskaya, I. Zizak, Direct observation of liquid pre-crystallization intermediates during the reduction of aqueous tetrachloroaurate by sulfide ions, Phys. Chem. Chem. Phys. 16 (10) (2014) 4538-4543.

[35] O. Gliko, W. Pan, P. Katsonis, N. Neumaier, O. Galkin, S. Weinkauf, P.G. Vekilov, Metastable liquid clusters in super- and undersaturated protein solutions, J. Phys. Chem. B 111 (12) (2007) 3106-3114.

[36] D. Kashchiev, P.G. Vekilov, A.B. Kolomeisky, Kinetics of two-step nucleation of crystals, J. Chem. Phys. 122 (24) (2005).

[37] Y. Li, V. Lubchenko, M.A. Vorontsova, L. Filobelo, P.G. Vekilov, Ostwald-like ripening of the anomalous mesoscopic clusters in protein solutions, J. Phys. Chem. B 116 (35) (2012) 10657-10664.

[38] A.L. Brazeau, N.D. Jones, Growth mechanisms in nanocrystalline lead sulfide by stopped-flow kinetic analysis, J. Phys. Chem. C 113 (47) (2009) 20246-20251.

[39] H.H. Teng, Thermodynamics of calcite growth: baseline for understanding biomineral formation, Science 282 (5389) (1998) 724-727.

[40] D. Pontoni, T. Narayanan, A.R. Rennie, Time-resolved SAXS study of nucleation and growth of silica colloids, Langmuir 18 (1) (2002) 56-59.

[41] Y. Sun, Controlled synthesis of colloidal silver nanoparticles in organic solutions: empirical rules for nucleation engineering, Chem. Soc. Rev. 42 (7) (2013) 2497-2511.

[42] X. Peng, J. Wickham, A.P. Alivisatos, Kinetics of II-VI and III-V colloidal semiconductor nanocrystal growth: "focusing" of size distributions, J. Am. Chem. Soc. 120 (21) (1998) 5343-5344.

[43] C. Bullen, P. Mulvaney, Nucleation and growth kinetics of CdSe nanocrystals in octadecene, Nano Lett. 4 (12) (2004) 2303-2307.

[44] W.W. Yu, L. Qu, W. Guo, X. Peng, Experimental determination of the extinction coefficient of CdTe, CdSe, and CdS nanocrystals, Chem. Mater. 15 (14) (2003) 2854-2860.

[45] M. Uehara, Z.H. Sun, H. Oyanagi, K. Yamashita, A. Fukano, H. Nakamura, H. Maeda, In situ extended X-ray absorption fine structure study of initial processes in CdSe nanocrystals formation using a microreactor, Appl. Phys. Lett. 94 (6) (2009) 17-20. 
[46] Z. Li, E. Dervishi, V. Saini, L. Zheng, W. Yan, S. Wei, A.S. Biris, X-ray absorption fine structure techniques, Part. Sci. Technol. 28 (2) (2010) 95-131.

[47] J. Wu, W. Gao, J. Wen, D.J. Miller, P. Lu, J.M. Zuo, H. Yang, Growth of Au on Pt icosahedral nanoparticles revealed by low-dose in situ TEM, Nano Lett. 15 (4) (2015) 2711-2715.

[48] B. Abécassis, F. Testard, O. Spalla, P. Barboux, B. Abécassis, Probing in situ the nucleation and growth of gold nanoparticles by small-angle X-ray scattering, Nano Lett. 7 (6) (2007) 1723-1727.

[49] D. Andreescu, T.K. Sau, D.V. Goia, Stabilizer-free nanosized gold sols, J. Colloid Interface Sci. 298 (2) (2006) 742-751. 